\title{
Research in the distance high resolution microwave seeker anti-ship missile target detection
}

\author{
Zhong zhi tong \\ Department of Missile Dalian Naval Academy, Dalian,116018,China \\ E-mail: mailbox1015@126.com
}

Keywords: Anti-ship; Microwave seeker; Target detection; Detector

\begin{abstract}
From high resolution is the development direction of microwave anti-ship missile seeker, low ends of the earth, distance target detection under the condition of high resolution and low resolution conditions has a very significant difference. On the analysis of the classical microwave seeker classical detection model and the optimal detector, on the basis of design a based on cycle accumulation of optimal secondary binary detector, analyzed the false alarm probability, detector parameter was optimized, finally adopts the field test data and the optimal detector performance comparison analysis, proved the practicability of the proposed detection method.
\end{abstract}

\section{Introduction}

Microwave anti-ship missile seeker, using new radar working system of FMICW can guarantee good transceiver isolation, and can achieve good distance high resolution performance, improve missile recognition and suppression of interference, is the important development direction of the future.

The adoption of sea-skimming missile terminal penetration flight, the sea clutter environment is evident in the wave peak effect, the distance of the distance and high resolution microwave seeker resolution cell length is far less than the ship geometry size, capture the target, target echo will be distribution in different distance resolution cell seeker, the formation of one-dimensional distance as the phenomenon of extended target distance.

Therefore, the low ends of the earth, distance target detection under the condition of high resolution and low resolution condition has the extremely significant difference between the two, need further analysis and research. Based on the classical detection model and on the basis of the optimal detector is proposed based on a cycle of accumulated more optimal secondary binary extended target detection method, and through the field test and parameter optimization of detection performance analysis.

\section{Detection model and the optimal detector}

In the microwave seeker has higher noise ratio conditions, sea clutter than inner noise receiver more influence on the target detection, so for target detection, temporarily don't consider the effect of internal noise receiver. Assumes that the coherent accumulation of FM cycles for P, receiving distance wave the width of the gate of $\mathrm{R}$ a distance resolution cell, and each distance unit contains only a echo after sampling data, extended target echo in which the data segment of length $\mathrm{L}$ a long period. Thus, echo data length is used to detect $\mathrm{H}=\mathrm{RP}$, target detection of hypothesis test model described as follows: 


$$
\left\{\begin{array}{l}
H_{0}: r_{t}=w_{t}, t=1,2, \ldots H \\
H_{1}:\left\{\begin{array}{l}
r_{t}=w_{t}+s_{t}, t \in \Omega_{s} \\
r_{t}=w_{t}, \quad t \in \bar{\Omega}_{s}
\end{array}\right.
\end{array}\right.
$$

Type, $\Omega_{s}$ data collection, to contain the target echo $\bar{\Omega}_{s}=\{1,2, \ldots, H\}-\Omega_{s} ; s_{t}, w_{t}$ Respectively extend target echo and high-resolution sea clutter, and both are independent of each other. Assume that echo $s_{t}$ signal amplitude obey parameters $A^{2}$ for Rayleigh fast relief model, phase in uniform distribution inside $[0,2 \pi]$;

Assume that the extent of high-resolution sea clutter $w_{t}$ to compound gaussian distribution, mutual $\left\{w_{t}\right\}$ statistical independence ${ }^{[1]}$,and $w_{t}=\tau_{t} g_{t}$. Among them, $g_{t}$ is the spot component for sea clutter, obey the zero mean gaussian distribution, its variance 1 ; The texture $g_{t}$ component for sea clutter, obey generalized chi-square distribution.

We can push it, the extent of sea clutter $\left|w_{t}\right|=\tau_{t}\left|g_{t}\right|$, with K distribution

$$
f_{|w|}\left(\left|w_{t}\right|\right)=\frac{2}{a \Gamma(v)}\left(\frac{\left|w_{t}\right|}{2 a}\right)^{v} K_{v-1}\left(\frac{\left|w_{t}\right|}{a}\right), v>0, a=\frac{b}{\sqrt{\pi}}>0
$$

The second type, $K_{v}(\square)$ is order modified Bessel function; For the parameters of the scale of the strength of sea clutter $a, v$ as the shape parameter, it determines the shape of the distribution of K, the smaller the value, the steeper the kurtosis of the distribution of $\mathrm{K}$, the longer the trailing, spike characteristics, the more obvious. Measurement results show that, $0.1 \leq v<\infty$ when the $\mathrm{K}$ degradation for Rayleigh distribution.

Moments of the distribution of $\mathrm{K}$ as follows:

$$
m_{k}=\frac{\Gamma(1+k / 2) \Gamma(v+k / 2)}{\Gamma(v)}(2 a)^{k}, k \geq 1
$$

In order to facilitate analysis, we fake dinghai clutter distribution parameters known or clutter data are used to estimate. In addition, for the microwave seeker, although the penetration process of missile and ship at the end of the high speed relatively games lead to target distance like walking across a distance unit, but because of the speed of ships is relatively low, using high-precision missile inertial device after motion compensation, the speed of information distance as scattering center moving across the range of problems can be solved effectively. In the following analysis process, therefore, is not considered as target distance walking across the range of problems.

Under the rule of Newman Pearson type (1) describe the hypothesis Test of the optimal detector for the Likelihood Ratio detector (Likelihood thewire Test, LRT), its expression is:

$$
\prod \frac{p_{1}\left(x_{t}\right)}{p_{0}\left(x_{t}\right)} \underset{H_{0}}{>} T_{h}
$$

Which $p_{1}\left(x_{t}\right)$ is under the assumption $H_{1}$ of the probability density distribution function, $p_{0}\left(x_{t}\right)$ is $x_{t}$ under the assumption $H_{0}$ of the probability density distribution function, $T_{h}$ for the corresponding threshold method.

Make $f\left(r_{t}\right)=\ln p_{1}\left(r_{t}\right)-\ln p_{0}\left(r_{t}\right)$ The type (4) can be represented as: 


$$
\sum_{t \in \Omega_{s}} f\left(r_{t}\right) \stackrel{H_{H_{0}}}{>} \ln T_{h}
$$

Type is the theory of microwave seeker binary detection on the optimal criteria.

\section{Based on more than two cycles accumulate the optimal secondary binary detection}

The detector design. The optimal secondary binary accumulation detection based on cycle idea is: first of all, through amplitude detector will high resolution distance like binarization, then using sliding window search way cycle of $\mathrm{M} / \mathrm{N}$ accumulation detection (airspace binary detection), and then during the week of $\mathrm{M} / \mathrm{N}$ accumulation detection (time-domain binary detection).

Because of the wave peak echo is sparse distribution and transient energy is very strong, the optimal secondary binary accumulation detection based on cycle use the sea clutter in the peak effect of this characteristic. First, binarization processing of echo amplitude detection is greatly weakened the wave peak echo, the effect of transient energy effectively restrain the interference of the wave peak;

Second, waves rush in time and space distribution is sparse, detector through its echo amplitude threshold probability is very low, after the time domain and airspace accumulation, sparse distribution waves rush to hardly more than a detection threshold, and ship target is a man-made object, has the stable shape structure, its echo in time and space is continuous, after accumulation of time domain, the airspace is easy to meet the threshold requirements. As a result, the optimal secondary binary detector based on cycle accumulation can greatly reduce the false alarm probability caused by the rush.

In addition, the adoption of the coherent accumulation detection methods, the detector does not need to doppler direction Vector (Steer Vector) information, simplifies the difficulty of the detector to achieve. Thus, using the optimal secondary cycles accumulate more binary detector for $\mathrm{K}$ distribution target detection under the clutter environment is a feasible technical way.

When using the optimal secondary binary accumulation detector based on cycle, makes $z_{p, l}=\left|r_{p, l}\right|$, among them, $r_{p, l}$ says the first the first $(p \in\{1,2, \ldots P\})(l \in\{1,2, \ldots U\})$ a cycle unit of distance sampling data. Assumes that the cycle of $\mathrm{M} / \mathrm{N}$ accumulation detection and $\mathrm{M} / \mathrm{N}$ accumulation detection parameters during weeks respectively: $M_{1} / N_{1}$ and $M_{2} / N_{2}$, the first threshold T amplitude detector. Testing process is divided into the following three steps ${ }^{[2]}$ :

Step 1: amplitude detection:

$$
\left[\begin{array}{l}
z_{p, l}< \\
<
\end{array}\right] \rightarrow d_{p, l}^{(1)}
$$

If $z_{p, l}>T$, then $d_{p, l}^{(1)}=1$, otherwise $d_{p, l}^{(1)}=0$. Through amplitude detection, can effectively weaken the peak echo waves the effect of transient energy.

Step 2: sliding window accumulation detection frequency modulation period. Unable to determine $\Omega_{s}$ the position in the entire data, therefore, the accumulation of cycle detection using sliding window search way, order $l_{w} \in\{0,1, \ldots, R-N+1\}$. Detector can be expressed as:

$$
\left[\sum_{l=l_{w}}^{l_{w}+N_{1}-1} d_{p, l}^{(1)}<M_{1}\right] \rightarrow d_{p, l_{w}}^{(2)}
$$

If $\sum_{l=l_{w}}^{l_{w}+N_{1}-1} d_{p, l}^{(1)} \geq M_{1}$, then $d_{p, l_{w}}^{(2)}=1$, otherwise $d_{p, l_{w}}^{(2)}=0$ 。 
Step 3: make $N_{2}=P$ for weeks during the accumulation of detection:

$$
\left[\sum_{p=1}^{P} d_{p, l_{w}}^{(2)}<M_{2}\right] \rightarrow d_{p, l_{w}}^{(3)}
$$

If $\sum_{p=1}^{P} d_{p, l_{w}}^{(2)} \geq M_{2}$, then $d_{p, l_{w}}^{(3)}=1$, otherwise $d_{p, l_{w}}^{(3)}=0$ 。

Finally, if $d_{p, l_{w}}^{(3)}=1$, argues that the sliding window corresponding position exists goals; On the other hand, argue that the sliding window target does not exist.

3.2 false-alarm probability analysis

Because the distance resolution cell within sea clutter is independent of each other, so the amplitude detection of false-alarm probability $P_{f 1}$ and false alarm probability of detection of cycle accumulation relations $P_{f, 2}$ can be expressed as:

$$
P_{f, 2}=\sum_{m=M_{1}}^{N_{1}} C_{m}^{N_{1}} P_{f, 1}^{m}\left(1-P_{f, 1}\right)^{N_{1}-m}
$$

Among them, the relationship with the threshold $\mathrm{T}$ and $P_{f, 1}$ is decided by the type:

$$
P_{f, 1}=\frac{2}{\Gamma(v)}\left(\frac{T}{2 a}\right)^{v} K_{v}\left(\frac{T}{a}\right)
$$

Total secondary binary detector false-alarm probability $P_{f}$ can be expressed as

$$
P_{f}=\sum_{m=M_{2}}^{N_{2}} C_{m}^{N_{2}} P_{f, 2}^{m}\left(1-P_{f, 2}\right)^{N_{2}-m}
$$

After cycle of $\mathrm{M} / \mathrm{N}$ accumulation detection, the detection probability of detector is:

$$
P_{d, 2}=\sum_{m=M_{1}}^{N_{1}} C_{m}^{N_{1}} P_{d, 1}^{m}\left(1-P_{d, 1}\right)^{N_{1}-m}
$$

Among them, $P_{d, 1}$ is for the single detection probability amplitude detector, can be represented as:

$$
P_{d, 1}=\int_{T}^{\infty} f_{z}\left(z_{t}\right) d z_{t}
$$

Type, the probability density function $z_{t}$ can be expressed by ${ }^{[3]}$ :

$$
f_{z}\left(z_{t}\right)=\int_{0}^{\infty} \frac{2 z_{t}}{A^{2}+\tau^{2}} \exp \left(-\frac{z_{t}^{2}}{A^{2}+\tau^{2}}\right) f_{\tau}(\tau) d \tau
$$

Thus, we can get the total detection probability $P_{d}$ of detector is as follows:

$$
P_{d}=\sum_{m=M_{2}}^{N_{1}} C_{m}^{N_{1}} P_{d, 2}^{m}\left(1-P_{d, 2}\right)^{N_{2}-m}
$$

From the above derivation, the detector of the detection probability $P_{d}$ and false alarm probability $P_{f}$ and detection threshold $\mathrm{T}$, the mathematical relationship between the parameters $M_{1}, M_{2}$ of complex, can only be analyzed by the method of numerical calculation to solve.

Using (9) (10) with type, type (11) for numerical simulation of the sea clutter shape parameters $v$, the relationship between the first detection threshold $\mathrm{T}$ and $M_{1}, M_{2}$ as shown in figure 1 , one of them. 
$a=1, P_{f}=10^{-6}, N_{1}=N_{2}=30, M_{1}=M_{2}$ The figure shows that when the parameter $M_{1}, M_{2}$ determination, detection threshold $\mathrm{T}$ as increases $M_{1}, M_{2}$; When the shape parameter determination, detection threshold $\mathrm{T}$ significantly reduced with the increase of. At the same time, because the detector adopted multiple cycles of coherent accumulation, detection threshold $\mathrm{T}$ is relatively low, generally not more than $23.0 \mathrm{~dB}$, is advantageous to the low signal noise ratio under the condition of the target signal detection.

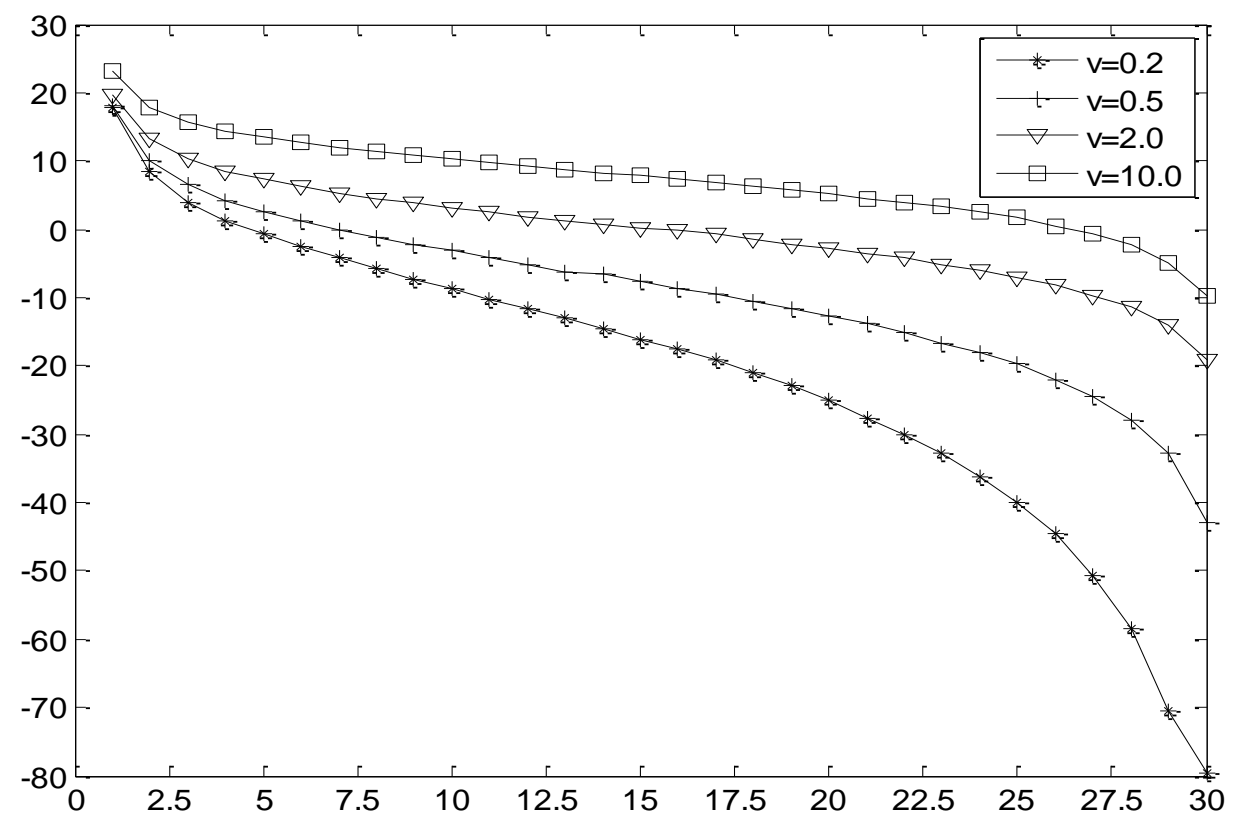

Fig. 1 Relationship between detection threshold T and M1, M2 for the detector

\section{Conclusion}

In this paper, the research and analysis shows that under the condition of the observed sea clutter based on cycle accumulation of optimal secondary binary detector can accurately detect the target, and the detection performance is superior to LRT detector.

\section{References}

[1] Ernesto Conte, Antonio De Maio, G. Ricci. GLRT-based adaptive detection algorithms for range-spread targets [J]. Signal Processing, IEEE Transactions on, 2001,49(7)

[2] S. D. Blunt, K. Gerlach, J. Heyer. HRR Detector for Slow-Moving Targets in Sea Clutter [J]. Aerospace And Electronic Systems, IEEE Transactions On, 2007,43(3)

[3] H. Griffiths. Sea Clutter: Scattering, the K Distribution and Radar Performance (Ward, K.D., et al.; 2006) [Book Review] [J]. Aerospace and Electronic Systems Magazine, IEEE, 2007,22(1)

[4]LI Zi fen, LI Xiang min, CHEN Jin zhu.Dynamic joint fire distribution method based on decentralized cooperative auction algorithm[J],Fire Control \& Command Control , 2012,37 (11): $: 50-52$

[5]NIAN Song lei, YAN Jian gang, CHEN Rong.The optimization method of antiship missile fire distribution based on dynamic programming[J],Ship Science And Technology, 2012,34 (7) : :110-113

[6]MA Liang, QING Zuo-sheng, ZHANG Lin.Formation comprehensive cooperative air defense 
multi-stage decision optimization modeling[J], Tactical Missile Technology, 2013,9 (5) :25-28

[7]LIU Wei dong, JIANG Qing shan, LI Yong.Fire distribution of the network centric ship-to-air missile based on earlier damage[J],Ship Science And Technology, 2011,33 (2):98-101

[8]CHEN Guo sheng, JIA Zi ying.Research on coordinated air defense firepower distribution model for warships[J], Command ControI\& Simulation, 2011,33 (12) :13-15 